dysphagia on either gastroscopy or barium studies. 10 (59\%) had an oesophageal dysmotility to account for their dysphagia - out of these one had achalasia; the other $9(53 \%)$ had oesophageal hypomotility with 7 having frequent hypotensive peristalsis and 2 having intermittent hypotensive peristalsis. 33\% of those with hypomotility had a normal Multiple Rapid Swallow test (MRS). 7 of the 17 patients (41\%) had normal oesophageal motility and 6 of these had reflux studies: $50 \%$ had GOR, 17\% had a hypersensitive oesophagus and the remaining $33 \%$ had normal studies. Only 2 patients $(12 \%)$ had hiatus hernias and both of these had oesophageal hypomotility and no GOR.

Conclusion This is the first study of upper GI physiology in patients with JHS and non-structural dysphagia. Oesophageal hypomotility is common in these patients and those patients with normal MRS are likely to show the best response to prokinetics. $50 \%$ of those with normal motility had pathological GOR and would benefit from high dose proton pump inhibitor therapy. The remainder had normal studies, suggesting hypersensitivity which may respond to treatment with neuromodulators such as amitriptyline. Only $12 \%$ of patients had a hiatus hernia, which is in contrast to previous studies. ${ }^{3}$ Further prospective studies are required to understand the pathophysiology and management options for dysphagia in JHS.

Competing interests None.

Keywords Dysmotility, Dysphagia, High Resolution Manometry, Joint Hypermobility, pH monitoring, Reflux.

\title{
REFERENCES
}

1. Castori M, et al. Am J Med Genet A 152A:556-64.

2. Zarate N, et al. Neurogastroenterol Motil 2009;22:252-e783.

3. Al-Rawi et al. Rheumatology (Oxford) 2004;43:574-6.

\section{Gl Physiology}

\section{PWE-122 \\ $\star$ DYSPHAGIA IN PATIENTS WITH THE JOINT} HYPERMOBILITY SYNDROME

doi:10.1136/gut.2011.239301.385

A Fikree, 1,* Q Aziz, J Jafari, ${ }^{1}$ R Grahame, ${ }^{2}$ D Sifrim ${ }^{1}$ 'Neurogastroenterology, Blizard Institute of Cell and Molecular Science, Barts and the London School of Medicine and Dentistry, London, UK; ${ }^{2}$ Rheumatology, University College Hospital, London, UK

Introduction The Joint Hypermobility Syndrome (JHS) is a relatively frequent inherited connective tissue disorder characterised by marked joint hyperextensibility and extra-articular manifestations. Recent work suggests that gastrointestinal (GI) symptom prevalence may be as high as $86 \%^{1}$ in these patients, and that many of them have evidence of GI dysmotility. ${ }^{2}$ Dysphagia is a symptom which is not uncommon in these patients, but which has never been formally studied. In general terms, dysphagia is either due to a structural abnormality of the oesophagus or a motility problem.

Methods A single-centre retrospective observational study was carried out to characterise the cause of non-structural dysphagia in patients with JHS. JHS patients who were referred to the upper GI physiology unit and who complained of dysphagia were identified. Their HRM and 24-h pH-metry traces were analysed to characterise oesophageal motility, presence of a hiatus hernia, lower oesophageal sphincter (LOS) pressure and presence of gastro-oesophageal reflux (GOR).

Results 17 patients with JHS and dysphagia were referred by the rheumatologists to our unit in 1 year: $76 \%$ female; age range: $12-58$. None had evidence of a structural cause for the 